\title{
Fatores de risco que favorecem a recaída no alcoolismo
}

\author{
Risk factors that favor the relapse in alcoholism
}

Armando M. Alonso Álvarez'

\section{RESUMO}

Objetivo: Existe uma prevalência estimada de 11,2\% de alcoolismo no Brasil. As tentativas de muitos alcoolistas para abandonar o consumo resultam fracassos e recaídas. A freqüência de recaída, segundo estudos, oscila entre 10\% e 30\%, assinalando-se diferentes causas. O objetivo da presente pesquisa é conhecer os fatores que favorecem as recaídas. Métodos: Aplicaram-se entrevistas e questionários "Fatores de Recaída" e "Razões para Beber" a 105 alcoolistas primários de ambos os sexos, e com predomínio de alcoolismo puro. Resultados: Os principais fatores de recaída são a pressão social, os conflitos inter-

\section{Palavras-chave}

Alcoolismo, recaída, fatores de recaída.

\section{Key-words}

Alcoholism, relapse, relapse factors. pessoais, os estados emocionais negativos, assim como a própria dependência fisiológica e psicológica. Conclusões: De maneira geral, os sujeitos apresentaram grande quantidade de fatores e razões que atuam simultaneamente favorecendo a recaída.

\section{ABSTRACT}

Objective: There is an estimate of $11.2 \%$ of alcohol prevalence in Brazil. The attempts of many alcoholics for abandoning the addiction result in failures and relapse. The number of relapse oscillates between 10 and 30\%, designating different causes. The objective of the present research is to know the factors that favor relapses. Methods: interviews and the questionnaires "Relapse Factors" and "Reasons for Drink" were applied, to 105 alcoholics of both sexes, with primary and typical alcoholism. Results: Initially, social demographics data are presented followed by a characterization of the information gotten in the interview and each questionnaire, which are integrated and submitted in discussion. Conclusions: the main factors are: the social pressure, the interpersonal conflicts, the negative emotional states as well as the proper physiological and psychological dependence. In general way, they had presented a great amount of factors and reasons that simultaneously act favoring the relapse.

1 Faculdade de Psicologia, Universidade da Havana. 


\section{INTRODUÇÃO}

Uma razão para esse estudo está na alta prevalência de alcoolismo no Brasil. No I Levantamento Domiciliar sobre o Uso de Drogas Psicotrópicas, envolvendo as 107 maiores cidades do país (2001), encontrou-se um número estimado de dependentes de álcool (11,2\%) (Carlini et al., 2002).

Uma segunda razão refere-se às conseqüências daninhas do álcool do ponto de vista biológico, psicológico e social, de amplo conhecimento (Alonso, 2006; Schuckit, 1991; Pinto de Queiroz, 1991). Como exemplo, Resende et al. (2002) assinalam que, no Brasil, o alcoolismo é a terceira causa de aposentadorias por invalidez e ocupa o segundo lugar entre os demais transtornos mentais.

O enfoque de risco assume que, quanto maior conhecimento sobre os eventos negativos, maior possibilidade de agir sobre eles com antecipação para evitá-los, mudando as condições que facilitem que um indivíduo ou grupo adquira a doença ou o dano.

A recaída é uma tentativa de parar ou diminuir o consumo de substâncias psicoativas, ou apenas o fracasso ao tentar atingir objetivos estabelecidos por um indivíduo, após um período definido (Jungerman e Laranjeira, 1999). É um regresso ao uso do álcool ou drogas, da mesma maneira que a pessoa usava antes de iniciar um programa de tratamento ou recuperação. Isto é, a pessoa que se tinha convertido em dependente e tinha eliminado o uso do álcool, retorna a ele (Knapp et al., 1994).

Para se considerar que houve uma recaída, exige-se que o paciente tenha conseguido, ao menos, dois meses de abstinência (www.yelop.galeon.com/craving.html).

A recaída é considerada uma parte do processo de reabilitação e não o final deste processo. Não significa que a pessoa tenha fracassado ou que não possa recuperar-se com o tempo.

Os estudos de seguimento (recuperação e recaída) indicam que aproximadamente um terço dos pacientes conseguem a abstinência permanente com sua primeira tentativa séria na recuperação. Um outro terço tem episódios breves de recaída, mas resultam, eventualmente, abstinência em longo prazo. O terço adicional tem recaídas crônicas, o que implica recuperações transitórias da adição química (Gorski et al., 1993). Dentro do método convencional, Marlatt e Rose referem a uma porcentagem de 70\% a 90\% de recaída dentro de três meses; Armor, Polich e Stambulk referem a uma incidência de 90\% de recaídas, após 18 meses (apud Reinecker, 1988). Segundo Parloff et al. (apud Gabbard, 1992), apenas 26\% dos alcoolistas tratados ainda permanecem abstinentes após um ano, em contraste com a taxa de remissão espontânea de 19\%.

De acordo com Knapp et al. (1994), Llama et al. (2003) existem diversas situações de risco que podem provocar as recaídas: a) emoções negativas, como a ansiedade, depressão, culpa etc., sendo estas o fator predominante que antecede ao primeiro consumo; b) pressão social com atribuições negativas, por exemplo, quando os amigos oferecem ou pressionam para consumir, quando o sujeito visita certas pessoas ou as recebe em casa, ir a festas etc.; c) manejo de situações difíceis, como enfrentar uma má notícia, terminar uma relação de casal, discussões no seio familiar etc.; d) manejo dos problemas físicos e psicológicos, como insônia, solidão, doenças próprias, problemas sexuais etc.; e) sintomas de abstinência unida a estímulos e incitações como precipitante.

Marlatt e Gordon $(1980,1985)$ acreditam que a recaída é influenciada pela inter-relação das situações ambientais condicionadas de alto risco, as habilidades para enfrentálas, o nível percebido de controle pessoal (auto-eficácia) e a antecipação dos efeitos positivos do álcool. Uma análise de 48 episódios revelou que a maioria das recaídas estava associada a três situações de alto risco: a) frustração e ira; b) pressão social; e c) tentação interpessoal.

A recaída não ocorre dentro de um vácuo. Há muitos fatores que contribuem para que ela ocorra, assim como também existem evidências ou sinais de advertência que indicam que um paciente pode estar no perigo do retorno ao abuso da substância (Gorski e Miller, 1993). A recaída não pode ser compreendida somente como o retorno real ao padrão do abuso da substância, mas também como o processo em que aparecem indicadores antes da volta do paciente ao uso da substância.

No mesmo sentido, Jungerman e Laranjeira (1999) destacam que a recaída deve ser encarada como um estado de transição, que pode ou não ser seguido de uma melhora. A recaída faz parte do processo de mudança e, muitas vezes, é essencial para que a pessoa possa aprender com a experiência e recomeçar de uma forma mais consciente. Neste sentido, é esclarecedor o modelo de estágios de mudança proposto por Prochaska e DiClemente (1982).

Marlatt e Gordon (1985) destacam que a recaída começa bem antes de reiniciar o consumo de álcool. As pessoas com respostas ineficazes a situações de alto risco experimentam falta de confiança, que, junto com as expectativas positivas quanto ao consumo de álcool, podem provocar um consumo deste, gerando sentimentos de culpa que, com o efeito positivo de álcool, the induzirão a seguir bebendo. Esses fatores são de duas classes: determinantes imediatos e antecedentes ocultos. Entre os determinantes imediatos estão as situações de alto risco, como estados emocionais negativos, situações de conflito interpessoal, situações de pressão social e estados afetivos positivos. Entre os antecedentes ocultos, estão o estilo de vida do paciente, os tipos de afrontamento ao estresse, o sistema de crenças etc., que mediam a resposta aos fatores imediatos. Depois acontece o deslize (consumo ocasional de álcool), que pode não conduzir a uma recaída, 
mas é um risco muito grande. Freqüentemente, depois do deslize, aparecem sentimentos de culpa que, com as expectativas positivas do álcool, levam ao consumo, que finaliza na recaída. Os autores têm descrito uma reação chamada efeito da violação da abstinência; esta reação se centra na resposta emocional que o sujeito tem diante do deslize. As pessoas que atribuem o deslize a uma incapacidade para se controlar recaíram com maior freqüência do que aqueles que pensam que têm sido ineficazes no manejo das situações. O objetivo deste estudo é avaliar os fatores de risco que favorecem a recaída nos sujeitos alcoolistas.

\section{MÉTODOS}

A amostra está composta por 105 sujeitos, com idade média de 42,9 anos. Todos os casos procedem de São João del Rei (SJDR) e de outras cidades do interior de Minas Gerais. Parte da amostra $(51,4 \%)$ estava internada na Clínica "Vila Esperança" SJDR, para tratamento antialcoólico, a outra parte $(49,6 \%)$ procede principalmente de Alcoólicos Anônimos e dos Postos de Saúde Familiar.

Como critérios de inclusão dos alcoolistas, utilizamos ambos os sexos, idade entre 20 e 70 anos, referência do sujeito ou instituição de ser alcoolista, existência de dano biológico, psíquico e/ou social produzido pelo álcool e perda de controle perante o tóxico, dois ou mais pontos no questionário "Cage" (a pontuação média alcançada foi de 3,50) e consentimento informado. Como critérios de exclusão: presença de transtornos psicóticos e de retardo mental moderado a profundo. presença de estado de embriaguez e não aceitar a avaliação.

Todos os alcoolistas são primários, predominando o alcoolismo típico (85\%).

\section{Avaliações}

Foram realizadas entrevistas de caráter semi-estruturado. A primeira entrevista teve como objetivo conferir a existência de alcoolismo e conhecer se há outra patologia de base. A segunda consta de cinco perguntas que indagam a história das recaídas, bem como os fatores que nelas influem.

\section{Instrumentos}

\section{Questionário de fatores de recaída}

Foi elaborado pelo autor, a partir, fundamentalmente, de listagens de eventos ou acontecimentos vitais apresentados por González (2004) e Alonso (1989). O questionário foi validado inicialmente a partir do critério de expertos e comprovada sua validade concorrente posteriormente na tese de Fernández (2006). Consta de 51 itens que incluem os fatores que podem provocar recaídas. Os sujeitos devem responder verdadeiro ou falso a estas perguntas e, ao final, assinalar os três fatores mais importantes.

\section{Questionário de razões para beber}

Este questionário foi elaborado por González (2004) e modificado pelo autor, com o objetivo de conhecer as alternativas escolhidas pelo sujeito acerca de sua conduta dependente. Consta de 13 razões, com possibilidade de agregar outras.

\section{Questionário Cage}

Este instrumento de medida consta de quatro perguntas que visam determinar o tipo de bebedor. Foi desenvolvido por Erwing e Rose em 1984 (Sáiz et al., 2002) e validado no Brasil por Masur e Monteiro (Pinto de Queiroz, 1991).

\section{Procedimentos}

Para a seleção dos sujeitos que recaíram e que estão sendo tratados em clínicas e em outras instituições de saúde, foram esperados sete dias, desde a data da sua internação até que tivessem sido eliminados os sintomas próprios da síndrome de abstinência. No caso dos sujeitos que freqüentam os Alcoólicos Anônimos ou que procedem de outro lugar, considerou-se que seu estado não interferiria no entendimento e na comunicação para a realização das entrevistas.

No início das entrevistas, deram-se as seguintes instruções aos sujeitos: "Estamos realizando um estudo sobre os motivos pelos quais as pessoas bebem e por que recaem no consumo de bebidas alcoólicas, a fim de propor um sistema de tratamento para evitar as recaídas". Explica-se que a pesquisa tem um caráter anônimo. Em seguida, é solicitado aos participantes que assinem um documento de consentimento informado.

\section{RESULTADOS}

Encontramos uma relação homem/mulher de 4:1. Uma análise por faixas etárias revela praticamente uma curva normal, observando-se nela a presença de menos sujeitos jovens e velhos. Em relação ao estado civil, observa-se um predomínio de sujeitos solteiros e separados/divorciados bem maior do que os casados. A análise da escolaridade mostra um baixo nível para a amostra total, em que 65\% têm analfabetismo ou primeiro grau incompleto, predominando as primeiras séries. Quanto à ocupação, há 27\% de desemprego. Em correspondência com a ocupação, se expressa um baixo salário. Quanto à convivência, 17\% mora sozinho e 83,8\% tem antecedentes familiares de alcoolismo.

Antes de passar a analisar os fatores que favorecem as recaídas, queremos destacar alguns dados do grupo estudado que resultam de interesse para compreender o fenômeno da recaída. A idade média de início do consumo foi de 15,1 anos, de aumento do consumo 23,9 anos, e de perda de controle 30,5 anos. Todos os sujeitos deixaram de beber completamente ao menos uma vez, a média foi de 2,29 vezes. 
Tabela 1. Variáveis sociodemográficas

\begin{tabular}{lr}
\hline Sexo & \\
Masculino & $85(81 \%)$ \\
Feminino & $20(19)$ \\
Faixas etárias & \\
$20-30$ & $11(10,4)$ \\
$31-40$ & $34(32,4)$ \\
$41-50$ & $35(33,3)$ \\
$51-60$ & $18(17,1)$ \\
Mais de 60 & $7(6,6)$ \\
Estado civil & \\
Solteiro & $42(40)$ \\
Casado / amasiado & $37(35,2)$ \\
Separado / divorciado & $21(20)$ \\
Viúvo & $4(3,8)$ \\
Escolaridade & \\
Analfabeto & $7(6,7)$ \\
Primeiro grau incompleto & $61(58,1)$ \\
Primeiro grau completo & $8(7,6)$ \\
Segundo grau incompleto & $12(11,4)$ \\
Segundo grau completo & $9(8,6)$ \\
Terceiro grau incompleto & $3(2,9)$ \\
Terceiro grau completo & $5(4,8)$ \\
Ocupação & \\
Empregado & $57(54,3)$ \\
Desempregado & $29(27,6)$ \\
Dona de casa & $6(5,7)$ \\
Estudante & $1(1,0)$ \\
Aposentado & $10(9,5)$ \\
Afastado & $2(1.9)$ \\
Renda mensal & \\
Menos de 1 salário mínimo & $37(35,2)$ \\
1 salário mínimo & $33(31,4)$ \\
2 salários mínimos & $16(15,2)$ \\
3 salários mínimos ou mais & $19(18,2)$ \\
Convivência & \\
Sozinho & $18(17,1)$ \\
Em companhia \\
Antecedentes familiares de alcoolismo \\
Sim & $87(82,8)$ \\
Não & \\
\hline & $88(83,8)$ \\
& $17(16,2)$ \\
\hline
\end{tabular}

Passamos a fazer uma análise dos fatores que favorecem a recaída no grupo de 105 alcoolistas que recaíram (internados e não internados). Este último grupo não está recebendo tratamento ambulatorial, somente assiste a Alcoólicos Anônimos um 11,4\% do total.

O método inicia-se com os dados provenientes da entrevista para posteriormente interpretar as informações obtidas nos questionários "Fatores de Recaída" e "Razões para Beber". Ao observar as perguntas da entrevista que foram analisadas qualitativamente, temos, em primeiro lugar, os fatores que influíram nas recaídas anteriores. Notamos que se destacam as dificuldades na área familiar (34,3\% dos sujeitos), tanto com a família de origem como a atual, em que existem discussões freqüentes, que levam em muitos casos à separação do cônjuge. Em segundo lugar, apreciamos a necessidade do consumo como expressão da dependência psicológica e fisiológica estabelecida (23,8\%); como importante, aparece também a influência negativa de amigos (19\%), que pode estar relacionada com a celebração e a festas (13,3\%). Quanto aos fatores que determinam a recaída atual, temos que os principais fatores são similares aos que influem nas recaídas anteriores.

Quanto ao por que os sujeitos que recaem não podem deixar de beber, aparece, em primeira ordem, o comportamento compulsivo ante o tóxico, a dependência ou craving; a seguir, a busca da bebida para a obtenção de prazer e alívio aos problemas que apresentam e, por último, a existência de diversos problemas familiares.

Na tabela 2 aparecem os fatores do questionário que, segundo os sujeitos, influíram na recaída, e vemos como fatores mais importantes (presente em $60 \%$ ou mais dos sujeitos) a necessidade de consumir e a falta de vontade associada, indicadora da dependência estabelecida; a pressão social de amigos bebedores, a assistência a lugares de consumo (festas, bares); a falsa crença de poder beber com controle; e, aparece como relevante, também, a existência de ansiedade e a autoestima diminuída. A média de fatores por sujeito é alta $(21,02)$.

Tabela 2. Questionário de "Fatores de Recaída": fatores mais freqüentes (em \%)

\begin{tabular}{lc}
\hline Fatores mais freqüentes & $(\mathrm{N}=105)$ \\
\hline 19 - Necessidade de beber & 78,1 \\
2 - Influência prejudicial de amigos bebedores & 73,3 \\
3 - Assistir a lugares onde consumia & 69,5 \\
7 - Existência de ansiedade & 68,6 \\
44 - Auto-estima baixa & 68,6 \\
4 - Crer que podia beber com controle & 65,7 \\
9 - Falta de vontade para deixar de beber & 61 \\
31 - Assistência a festas & 60 \\
\hline
\end{tabular}

A quantidade de fatores que os sujeitos assinalam como provocadores da recaída é alto. Vemos que $54 \%$ de sujeitos apresentam mais de 20 fatores, o que pode ser índice da multiplicidade de influências, tanto pessoais como "externas", que estão incidindo de maneira simultânea.

A seguir, faz-se uma análise que se vincula com a tabela 1, já que se pediu ao sujeito que nomeasse os três fatores mais importantes que, a seu juízo, favoreciam neles a recaída. Isso faz que a dispersão seja muito grande, já que são escolhidos entre 51 itens. Aparece de maneira relevante a influência negativa de amigos bebedores (40 sujeitos), o que pode ser interpretado como atribuir ao outro a culpa, ou bem, que a recaída se dá por causa da inter-relação que se estabelece com outros. Na seqüência, ainda que em menor medida, aparece a necessidade de beber (20) unida ao pobre controle no consumo (20). Destaca-se, como na entrevista, a existência de problemas importantes na área familiar, como ausência de apoio (18), separações do casal (12) e morte de familiares (12). Merecem ser interpretados aqueles estados emocionais negativos, como ansiedade (11) e depressão (11).

Em relação ao Questionário "Razões para Beber", que complementa a análise do questionário anterior, vemos que as razões (fatores) mais importantes foram: alegrar-se e rela- 
xar-se, como estados emocionais positivos que se procura por meio da bebida, bem como esquecer preocupações e eliminar ansiedade, como estados emocionais negativos, que se trata de eliminar pelo consumo, os quais estão em estreita inter-relação. Todos os fatores foram selecionados por mais de $40 \%$ dos sujeitos. Cerca de $60 \%$ dos sujeitos que recaíram marcaram mais de sete razões para beber e $88 \%$ mais de quatro razões. A média de razões foi 7,30.

Tabela 3. Questionário "Razões para beber"

\begin{tabular}{lc}
\hline & Recaídas (N=105) \\
\hline Acompanhar as refeições & $43(40,9 \%)$ \\
Celebrar, comemorar & $51(48,8 \%)$ \\
Alegrar-se & $81(77,1 \%)$ \\
Esquecer preocupações & $68(64,7 \%)$ \\
Sentir-se mais seguro & $52(49,5 \%)$ \\
Sentir-se sociável & $56(53,3 \%)$ \\
Relaxar-se & $81(77,1 \%)$ \\
Ser aceito no grupo & $59(56,2 \%)$ \\
Ser valente & $36(34,3 \%)$ \\
Acompanhar a vida sexual & $39(37,1 \%)$ \\
Vencer temores & $58(55,2 \%)$ \\
Eliminar a depressão & $67(63,8 \%)$ \\
Estar menos ansioso & $75(71,4 \%)$ \\
Outras razões & $7(6,7 \%)$ \\
\hline
\end{tabular}

\section{DISCUSSÃO}

A pesquisa realizada por Fernández (2006), com orientação do autor da atual pesquisa, encontrou na entrevista, na amostra cubana de 25 casos, que os fatores fundamentais para as recaídas anteriores foram: a pressão social e a influência negativa de amigos (40\% de sujeitos), a existência de solidão e estar distante da família (24\%), os conflitos com a parceira (16\%), a dependência (10\%) e a depressão (8\%). Neste caso aparece a influência de outros para beber como primeiro fator, o que a diferencia da presente pesquisa. Não obstante, existe uma coincidência nos principais indicadores pelos quais se recai.

$\mathrm{Na}$ recaída atual, segundo Fernández, os sujeitos continuam destacando as más influências (32\%), a existência de conflitos na família (16\%), não saber enfrentar certas situações (12\%), morte de familiares (4\%) e, aparece como fator mais relevante e novo, a falta de vontade (36\%).

Existem diferenças importantes entre os grupos, no sentido que os alcoolistas brasileiros fazem referência aos fatores dependência/compulsão e festas/carnaval como fatores de recaída, que não aparecem na amostra cubana.

Comparando nossos resultados no Questionário de recaída, com os fatores encontrados por Fernández (2006) no mesmo instrumento, se evidencia bastante coincidência, mas novamente aparece relevante na amostra brasileira e para nada na cubana, a necessidade de beber e a falta de vontade para deixar de beber, como fatores importan- tes para a recaída. Esses fatores próprios da dependência fisiológica e psicológica têm sido evidenciados por outros especialistas (Marlatt e Gordon, 1993).

Rigotto e Gómez (2000) pesquisaram as situações favorecedoras de recaída em um grupo de jovens consumidores de álcool e outras drogas, que tinham recaído nos últimos seis meses, encontrando: falta de apoio familiar, falta de acompanhamento apropriado, envolvimento com antigos amigos usuários, necessidade de aprovação social e frustrações diante de circunstâncias adversas.

$\mathrm{Na}$ pesquisa com 20 alcoolistas (em tratamento e abstinentes), o motivo mais declarado para o início do consumo do álcool foi também a influência de amigos e colegas (65\%) (Correa e Pardo, 2004). A influência negativa do grupo social é apontada como um dos fatores que mais contribuem para a recaída. A longa história de consumo intenso de álcool influencia na construção de uma auto-imagem negativa relacionada ao poder de comunicação dos dependentes, resultado de uma gradativa desvalorização de seus discursos, ao longo dos anos. Dessa forma, os alcoólicos se sentem derrotados e impotentes, e, invariavelmente, sofrem de baixa auto-estima. Durante o período de consumo, os dependentes restringem suas relações sociais a pessoas que também fazem uso do álcool (Carrol, 2002).

Segundo Makken (1999), a personalidade do adicto caracteriza-se por um enfraquecimento do eu, da vontade e aumento da perda do controle, por um vazio existencial com a falta de uma atividade que Ihe proporcione satisfação, pelo desenvolvimento de rituais adictivos, a partir dos quais se reafirma em suas novas crenças e valores (estilo de vida adictivo), pelo quebrantamento emocional etc.

Para Caballo (2003), independentemente da causa do déficit em habilidades, o abuso de substâncias psicoativas pode servir como um meio para enfrentar a vida diária e/ou as fortes pressões externas.

Os trabalhos de Litman (1986) e de Laranjeira e Souza (citado por Correa e Pardo, 2004) demonstraram uma relação direta entre a percepção de auto-eficácia dos alcoolistas nas condutas de afrontamento e os resultados posteriores. Aqueles sujeitos que recaíam entre os 6 e 15 meses percebiam mais situações como fatores de risco e sentiam menos controle sobre seus estados emocionais negativos, ao mesmo tempo que eram mais vulneráveis aos acontecimentos externos. Não acreditavam que suas condutas de afrontamento eram efetivas e não observavam relações entre as situações que percebiam como perigosas tampouco sua habilidade para lidar com elas. Os sujeitos que não recaíram durante este período percebiam seus pensamentos como positivos e evitavam certas situações, como condutas de afrontamento efetivas, bem como percebiam menos situações perigosas para continuar sua abstinência.

Em pesquisa de comparação (Fernández, 2006), também se encontrou grande número de fatores, apresentando de 5 a 9 fatores (44\% de sujeitos), de 10 a 14 (40\%) e de 15 a 20 (16\%). Ne- 
nhum caso cubano apresentou mais de 20 fatores, o que contrasta com a amostra brasileira, em que mais de $54 \%$ dos sujeitos apresentaram mais de 20 fatores. Isso retrata o grande número de contingências ambientais e pessoais a que estão submetidos os alcoolistas e como é difícil lidar com elas no tratamento.

Como no "Questionário de fatores de recaída", no "Questionário de razões para beber", a amostra cubana apresentou menos razões para beber: 7 a 9 razões (36\%), de 4 a 6 (60\%) e de 1 a 3 (4\%). Este fato leva a considerar que o alcoolismo e em particular a recaída estão associados, no Brasil, a grande número de situações externas e internas, de diferente natureza.

\section{CONCLUSÕES}

Os fatores favorecedores encontrados nestes estudos seriam: a) pressão social: influência prejudicial de amigos bebedores, ir a lugares onde se consume, a festas e celebrações; b) dependência (craving): necessidade de beber, beber com controle e falta de vontade para deixar de beber; c) conflitos interpessoais, fundamentalmente com a família (separações, brigas, falta de apoio e harmonia familiar); d) estados emocionais negativos (ansiedade, depressão, raiva). Existiu uma média de 21 fatores no Questionário de Fatores que têm favorecido a recaída nos sujeitos. Aproximadamente 54\% dos sujeitos apresentaram mais de 20 fatores de recaída, o que é índice da multiplicidade de influências, tanto pessoais como "externas" que estão incidindo de maneira simultânea. Este grupo citou, no Questionário Razões para Beber, como mais relevante: consumir para alegrar-se e relaxar-se (procurando estados emocionais agradáveis), e esquecer preocupações e eliminar ansiedade (evitando estados emocionais desagradáveis). Aproximadamente $62 \%$ dos sujeitos que recaíram escoIheram mais de sete razões para beber. A alta quantidade de razões e fatores que favorecem a recaída indica a dificuldade de considerar todas estas variáveis no tratamento e a dificuldade que resulta a reabilitação.

Potenciais conflitos de interesse: Instituição na qual o trabalho foi produzido: Universidade Federal de São João Del Rei (UFSJ) - Pesquisador visitante por Fundação de Amparo à Pesquisa do Estado de Minas Gerais (Fapemig).

\section{REFERÊNCIAS}

Alonso A. Mitos y realidades sobre el alcoholismo [acesso em 2006 Nov 6]. Disponível em: http://www.monografias.com/trabajos39/mito-realidad-alcoholismo/mito-realidadalcoholismo.shtml/

Alonso A. Papel de la personalidad anómala en el origen de la neurosis y de sus diversas formas clínicas [tesis de doctorado]. Facultad de Psicología, Universidad de La Habana; 1989.
Caballo V. Manual de avaliação e treinamento das habilidades sociais. São Paulo: Ed. Livraria Santos, 2003.

Carlini E, Galduróz J, Noto A, Nappo S. I levantamento domiciliar nacional sobre o uso de drogas psicotrópicas no Brasil. Escola Paulista de Medicina. Departamento de Psicobiología. Centro Brasileiro de Informações sobre Drogas Psicotrópicas (CEBRID), São Paulo; 2002.

Carrol KM. Las terapias conductuales basadas en la ciencia para la dependencia de drogas. Psiquiatría y Salud Integral, 2,1, 46-53. 2002

Correa LE, Pardo MB. Avaliação de habilidades sociais em dependentes alcoólicos. Boletim de Psicologia, vol. LIV, no. 120: 87-104, 2004

Cummings C, Gordon JR, Marlatt GA. Relapse: prevention and prediction. In: Miller WR, editor. The addictive behaviors. New York: Pergamon; 1980.

Fernández S. Factores de recaída en el alcoholismo. Una propuesta de tratamiento [trabajo de diploma para optar por el Título de Licenciado en Psicología]. Facultad Psicología, Universidad Habana; 2006.

Gabbard G. Psiquiatria psicodinâmica na prática clínica. Porto Alegre: Artes Médicas, 1992.

González R. Alcoholismo. Abordaje integral. Ed. Oriente, Santiago de Cuba.: Ed. Oriente, 2004.

Gorski TT, Kelley JM, Havens L. An overview of addiction relapse and relapse prevention. In: Relapse prevention and the substance-abusing criminal offender (An executive briefing) (Technical Assistance Publication Series 8). Rockville, MD: (enter for Substance Abuse Treatment, 1993.

Gorski T, Miller M. Counseling for relapse prevention. Independence, MO: Herald House/Independence, 1982.

Jungerman F, Laranjeira R. Entrevista motivacional: bases teóricas e práticas. J Bras Psiquiatr, 48 (5): 197-207, 1999

Knapp P, Bertolote JM et al. Prevenção da recaída: um manual para pessoas com problemas pelo uso de álcool e das drogas. Porto Alegre: Artes Médicas, 1994

Litman GK. Alcoholism survival: the prevention of relapse. In: Miller WR, Heather M, editors. Treating addictive behaviors: processes of change. 391-405, New York: Plenum Press).

Luz E. Álcool: uso nocivo e dependência. In: Taborda JGV, Prado-Lima PP, Busnello EDA. Rotinas em psiquiatria. pp. 83-99. Porto Alegre: Artes Médicas 1996

Makken C. La personalidad adictiva. México: Diana, 1999.

Marlatt AG, Gordon JR. Determinants of relapse: Implications of the maintenance of behavior change. In: Davidson P0, Davidson, SM, editors. Behavioral medicine: changing health lifestyle. New York: Brunner/Mazel: 410-452, 1980.

Marlatt AG, Gordon GR. Relapse prevention. Maintenance strategies in the treatment of addictive behaviors. New York: Guilford, 1985.

Pinto de Queiroz J. Função, disfunção e controle da mente humana. Fundamentos de psiquiatria para a clínica diária. Salvador, Bahia: Universitária Americana; 1991.

Prochaska J0, DiClemente CC. Transtheoretical therapy: towards a more integrative model of change. Psychotherapy: Theory, Research and Practice, 19:276-288, 1982

Reinecker H. Alcoolismo. In: Lettner, HW, Rangé BP. Manual de psicoterapia comportamental: 94-103. Ed. Manole Ltda., 1988.

Resende G, Amaral V, Bandeira M, Gomide A, Andrade E. Análise da prontidão para o tratamento em alcoolistas em um centro de tratamento. Revista Psiquiatria Clinica, 32 (4): 211-217, 2005.7.

Rigotto S, Gómes W. Contextos de abstinência e de recaída na recuperação da dependência química [acesso em 2007 May 17]. Disponível em: www.adroga.casadia.org/recuperacao/ abstinencia-e-recaida.htm+fatores/

Sáiz PA, G-Portilla MP, Paredes MB. Bascarán MT, Bobes J. Instrumentos de evaluación en el alcoholismo. Monografía alcohol, España. Revista Adicciones vol. 14 suplemento 1, España, 2002.

Schuckit M. Abuso de álcool e drogas. Uma orientação clínica ao diagnóstico e tratamento. Porto Alegre: Artes Médicas, 1991.

Vasconcelos A, Neto JH. Descrição de uma população de alcoolistas internados em Maceió. Jornal Brasileiro Psiquiatria, 46(6): 325-329, 1997.

Site

www.yelop.galeon.com/craving.html. (Acessado em 7 de dezembro de 2006). 